Costa, J.F.F.; Deprá, P.P.; Abiko, R.H.; Weber, V.M.R.; Fernandes, D.Z.; Zavalla, S.A.; Borges, P.H. (2021) Effects of the Standards Breath by Strokes in the Crawl Swimming. Revista Internacional de Medicina y Ciencias de la Actividad Física y el Deporte vol. 21 (82) pp. 223-234 Http://cdeporte.rediris.es/revista/revista82/artefecto1254.htm

DOI: https://doi.org/10.15366/rimcafd2021.82.002

\title{
ORIGINAL
}

\section{EFECTO DE LA CANTIDAD DE RESPIRACIONES POR BRAZADAS EN EL NADO CRAWL}

\section{EFFECTS OF THE STANDARDS BREATH BY STROKES IN THE CRAWL SWIMMING}

Costa, J.F.F.'; Deprá, P.P. ${ }^{1}$; Abiko, R.H. ${ }^{2}$; Weber, V.M.R. ${ }^{3}$; Fernandes, D.Z. ${ }^{3}$; Zavalla, S.A. ${ }^{4}$ y Borges, P.H. ${ }^{1}$

1 Departamento de Educação Física, Universidade Estadual de Maringá (Brasil) jonathan.mga@gmail.com, ppdepra@gmail.com, pauloborges.uem@gmail.com

2 Programa de Pós-Graduação em Promoção da Saúde, Unicesumar (Brasil) rafaelabiko@gmail.com

3 Programa de Pós-Graduação Associado em Educação Física UEM/UEL. Universidade Estadual de Londrina (Brasil) viniciusweber@uel.br, danizafe@gmail.com

${ }^{4}$ Estudiante de Magíster en Actividad Fìsica y Salud, Universidad Católica del Maule (Chile) sebarenazavalla@gmail.com

Código UNESCO / UNESCO Code: 2406.04 Biomecánica / Biomechanics; 6106.09 Procesos de Percepción / Perception Processes

Clasificación Consejo de Europa: 3. Biomecánica del Deporte/Sport Biomechanics of Sport;12. Aprendizaje Motor/Motor Learning

Recibido 10 de junio de 2019 Received June 10, 2019

Aceptado 8 de septiembre de 2019 Accepted September 8, 2019

\section{RESUMEN}

El objetivo de este estudio es investigar los efectos de la cantidad de respiraciones realizadas por brazada en el desempeño del nado crawl. Participaron del estudio 32 nadadores de ambos géneros (37.12 \pm 12.51 años). Los sujetos fueron grabados nadando 25 metros en el estilo crawl a velocidad máxima. No hubo diferencia significativa entre el tiempo de duración de la prueba y la velocidad promedio de los patrones respiratorios, sin embargo, los ciclos de brazadas de los patrones R2 y R3 presentaron mejores resultados $(p<0.05)$. La frecuencia de brazada de los patrones respiratorios R2 (0.64 ciclo/s) y R3 (0.65 ciclo/s) fueron menores $(p<0.04)$ en comparación con los patrones R4 y R5. El IMC aumenta el tiempo necesario para el rendimeinto óptimo de la prueba ( $\beta=$ 0.39 ). Se concluye que el patrón respiratorio $\mathrm{R} 2$ fue el más eficaz para nadadores aficionados. 
PALABRAS CLAVE: natación, nado crawl, respiración.

\section{ABSTRACT}

Objective: The aim of the study was to analyze the amateur's performance of crawl swimming in different types of respiratory standards. The sample was composed of 32 swimmers of both sexes (37.12 \pm 12.51 years). They were filmed at 25 meters of crawl swimming at maximum speed. There were no significant differences in the duration of the test and speed between respiratory standards. However, the stroke cycle of the R2 and R3 standards presented better results $(p<0.05)$. The Body Mass Index increased the time necessary for the completion of the test $(\beta=0.39)$. In conclusion, the R2 standard was more effective for amateurs.

KEY WORDS: swimming, crawl stroke, breath.

\section{INTRODUCCIÓN}

La natación es una modalidad deportiva presente en una porción significativa de la población a nivel mundial, siendo utilizada en su mayoría como un deporte de rehabilitación de pacientes con algún tipo de lesión física o muscular (da Silva, de Oliveira, \& Conceição, 2005). Siendo este uno de los factores esenciales de la popularidad de este deporte en Brasil y por consecuencia la profundización y aprendizaje de su variedad de estilos como el crawl, espalda, pecho y mariposa (Massaud \& Côrrea, 2008).

El crawl es una variedad o estilo dentro de este deporte, el cual necesita de varios factores o movimientos para su correcta ejecución, tales como: la brazada, que es el principal elemento de propulsión, la patada que es el movimiento que le entrega estabilidad al cuerpo durante el movimiento, el tronco, el cual ayuda a conservar la alineación lateral del cuerpo y la respiración, que esencialmente nos proporciona el oxígeno necesario (Marcos Roberto Apolinário, 2010). La identificación de factores biomecánicos como la frecuencia de brazada, la velocidad media y el índice de brazada son importantes, ya que ejercen gran influencia sobre el arrastre y desarrollo de la fuerza propulsora (de Souza Castro, Guimarães, Moré, Lammerhirt, \& Maques, 2005; Toussaint \& Hollander, 1994).

En cuanto a la respiración, se entiende que la menor cantidad de esta por ciclos de brazadas ocasionará un mejor desempeño del nadador, gracias al efecto de arrastre activo ocasionado por los movimientos propios del cuerpo durante la respiración, promoviendo un aumento del tiempo en el desplazamiento de los nadadores (Apolinario et al., 2012; Marcos Roberto Apolinário, 2010).

A pesar de estas evidencias, la mayoría de los resultados encontrados en la literatura fueron basados en atletas de alto rendimiento, los cuales poseen altos niveles de habilidad, con capacidad de adaptarse rápidamente a cambios en el patrón respiratorio (M R Apolinário et al., 2007). Además, se vuelve fundamental 
en los profesionales o docentes de Educación Física la búsqueda de investigaciones o estudios que tengan que ver con este tema, como si la menor cantidad de respiraciones por ciclos de brazadas ocasiona un mejor desempeño en nadadores iniciales o temas similares a ese. Estos datos les pueden ser de utilidad en la planificación y ejecución de las clases, en cuanto a sus objetivos y contenidos a ser trabajados.

\section{OBJETIVOS}

Es por ello que los objetivos de esta investigación son los siguientes: I) comparar el desempeño del nado crawl en nadadores aficionados con diferentes patrones respiratorios y grupos de desempeño; II) identificar las contribuciones relativas de variables antropométricas y biomecánicas sobre el desempeño del nado crawl.

\section{MATERIAL Y MÉTODOS}

\section{Participantes}

Participaron del estudio 32 nadadores aficionados de ambos sexos (37.12 \pm 12.51 años) pertenecientes a un proyecto de extensión de una universidad pública en el estado de Paraná. Se utilizaron los siguientes criterios de inclusión: (1) tener entre 18 y 60 años; (2) Practicar natación por lo menos 2 veces por semana. Los índices de exclusión fueron: (1) presentar alguna lesión musculoesquelética; (2) Tener menos de 4 meses de práctica en la natación. El estudio fue aprobado por el comité de ética e investigación local (Dictamen $n^{\circ}$ 2.927.011) y, tras consentimiento, se invitó a los voluntarios a firmar el consentimiento informado necesario para el estudio.

\section{Procedimientos}

El nado de cada voluntario se registró mediante grabaciones de video con una cámara Samsung ${ }^{\circledR}$ Canon. El investigador registró todo el recorrido de la prueba, desde el borde lateral externo de la piscina hasta el final de ella.

Los participantes realizaron un calentamiento de 10 minutos según el patrón adoptado en el estudio de Apolinário (2010). Asumiendo que esta cantidad de minutos es suficiente para provocar el aumento de la frecuencia cardíaca y activar los principales músculos involucrados en la actividad a realizar.

Posterior al calentamiento, se entregaron las instrucciones técnicas del nado crawl para su correcta ejecución, la posición inicial del nadador debía ser dentro de la piscina, con el cuerpo en la horizontal y con los pies en la pared. Después de la señal realizada por un silbato, el nadador inició su nado crawl, recorriendo 25 metros en ritmo de prueba, en la condición de respiración solicitada por el experimentador. Cada participante ejecutó los siguientes patrones respiratorios: dos brazadas para respirar (crawl R2); Tres brazadas para respirar (crawl R3); cuatro brazadas para respirar (crawl R4); cinco brazadas para respirar (crawl R5) y respiración libre (crawl $\mathrm{RL}$ ). En las respiraciones pares el nadador eligió su lado 
de preferencia para la respiración. El orden de los patrones respiratorios por nadador fue definido de forma aleatoria por medio de sorteo en una planilla excel.

Al finalizar cada serie de 25 metros, el sujeto se cambiaba a un carril diferente para dar paso al siguiente participante. Después de que el nadador ejecutara el nado con el primer patrón respiratorio solicitado por el investigador, se le otorgaba un descanso de aproximadamente 5 minutos, con el fin de minimizar el efecto de la fatiga. Según Maglischo \& do Nascimento (1999), en pruebas de $25 \mathrm{~m}$ existen dos causas para la fatiga: el descenso de las reservas de creatina fosfato muscular (CP) y la incapacidad de hacer operativo el metabolismo anaeróbico con rapidez. Después de los 5 minutos, los procedimientos para el segundo patrón se reiniciaron y así sucesivamente hasta el quinto patrón respiratorio.

\section{Variables del estudio}

Para evaluar el desempeño del nado crawl, se utilizaron variables propuestas por Caputo, De Lucas, Greco, \& Denadai, (2008) y Hay (1981), Ciclo de brazadas, correspondiente al total de brazadas completas efectuadas durante la prueba; Longitud de brazada (LB), que es la distancia horizontal media recorrida durante la ejecución de un ciclo completo de los brazos; Frecuencia de brazada (FB), siendo el número medio de ciclos completos de los brazos ejecutados por segundo; Velocidad media $(\mathrm{Vm})$, velocidad con que el nadador se mueve a través del agua; Índice de brazada (IB), obtenido a partir del producto entre la velocidad media y la longitud de brazadas. Según Caputo et al., (2008) cuanto mayor sea el índice de brazada, la técnica del nadador será más efectiva.

Las medidas antropométricas de masa corporal y estatura fueron obtenidas por medio de una balanza digital calibrada, con carga máxima de $180 \mathrm{~kg}$ y escala de $0.1 \mathrm{~kg}$ y un estadiómetro de madera con escala de $0.1 \mathrm{~cm}$, siguiendo procedimientos descritos por Guedes (2006).

\section{Análisis estadístico}

Para el análisis estadístico se utilizó la prueba de Shapiro-Wilk para verificar la normalidad de los datos. La prueba ANOVA de medidas repetidas fue empleada para la comparación de las variables dependientes "duración", "ciclo de brazada", "longitud de brazada", "frecuencia de brazada", "velocidad media" e "índice de brazada" entre los patrones respiratorios (" R2, R3, R4, R5 y RL), seguido del post-hoc de Bonferroni para identificar dónde estaban las diferencias entre cada una de ellas.

En consecuencia, se utilizó un procedimiento estadístico multivariado denominado Análisis de Cluster (Marôco, 2014), a través del método no jerárquico K-means, con el fin de clasificar a los nadadores en dos grupos de rendimiento técnico (cluster 1 - peor desempeño técnico: $n=21$, cluster 2 - mejor rendimiento técnico: $n=11$ ), donde la variable "índice de brazada" fue utilizada en el modelo. A partir de la creación de los grupos, se recurrió a la prueba t para 
muestras independientes para comparar indicadores de tamaño corporal, edad y frecuencia en entrenamientos de ambos grupos.

Por último, se realizó un análisis de regresión lineal múltiple para estimar las contribuciones relativas de las variables antropométricas y técnicas sobre el tiempo (segundos) de los nadadores en las diferentes condiciones de nado, controlando por sexo, edad y frecuencia semanal de entrenamientos.

\section{RESULTADOS}

La tabla 1 presenta las características antropométricas de los practicantes de natación del proyecto de extensión:

Tabla 1. Características antropométricas de los practicantes de natación no competitiva.

\begin{tabular}{lcccc}
\hline & Media & Desviación estándar & Mínimo & Máximo \\
\hline Edad (años) & 37.12 & 12.51 & 18.00 & 60.00 \\
Estatura (m) & 1.71 & 0.10 & 1.46 & 1.89 \\
MC (kg) & 74.30 & 12.17 & 55.90 & 100.20 \\
IMC (kg/m $\left.\mathbf{m}^{2}\right)$ & 25.15 & 3.24 & 20.99 & 36.21 \\
\hline
\end{tabular}

Nota: $\mathrm{m}=$ metros; $\mathrm{kg}=$ kilogramos.

La tabla 2 presenta la comparación entre la "duración", el número de ciclos de brazadas, la frecuencia de brazada, la velocidad media y el índice de brazada entre los patrones respiratorios R2, R3, R4, R5 y RL. 
Rev.int.med.cienc.act.fís.deporte - vol. 21 - número 82 - ISSN: 1577-0354

Tabla 2. Comparación entre duración, número de ciclos de brazadas, frecuencia de brazada, velocidad media e índice de brazada entre los patrones respiratorios de los sujetos estudiados.

\begin{tabular}{|c|c|c|c|c|c|}
\hline & $\mathbf{R 2}$ & R3 & R4 & R5 & $\mathbf{R L}$ \\
\hline & Media (DP) & Media (DP) & Media (DP) & Media (DP) & Media (DP) \\
\hline Duración (s) & $20.93(4.23)$ & $21.31(4.08)$ & $21.12(3.98)$ & $21.37(4.40)$ & $21.09(4.34)$ \\
\hline Velocidad media $(\mathrm{m} / \mathrm{s})$ & $1.23(0.23)$ & $1.21(0.23)$ & $1.22(0.23)$ & $1.21(0.24)$ & $1.23(0.24)$ \\
\hline Número de Ciclos de Brazadas (ciclos) & $13.50(3.02) \mathrm{bcd}$ & $13.78(2.79)^{b c d}$ & $14.21(3.00)$ & $14.40(3.03)$ & $14.65(3.00)$ \\
\hline Longitud de Brazadas (m/ciclo) & $1.95(0.50)$ bcd & $1.88(0.39)^{c d}$ & $1.84(0.45)$ & $1.81(0.40)$ & $1.77(0.35)$ \\
\hline Frecuencia de Brazadas (ciclo/s) & $0.64(0.09)^{d}$ & $0.65(0.09)^{d}$ & $0.67(0.10)$ & $0.68(0.10)$ & $0.70(0.10)$ \\
\hline Índice de Brazada (m2/ciclo*s) & $2.50(1.03) \mathrm{bcd}$ & $2.35(0.85)$ & $2.33(0.93)$ & $2.27(0.88)$ & $2.24(0.81)$ \\
\hline
\end{tabular}

Nota: ${ }^{a}$ Diferencia para R3; ' Diferencia para R4; ' ${ }^{2}$ iferencia para R5; ${ }^{\mathrm{d} D i f e r e n c i a ~ p a r a ~ R L . ~} \mathrm{~s}=$ segundos; $\mathrm{m}=\mathrm{metros.}$ 
No se observaron diferencias significativas entre las variables "duración" y "velocidad media" en los patrones respiratorios, lo que muestra similitudes en el desempeño. Sin embargo, el ciclo de brazadas presentó diferencias entre los patrones R2 y R3 con el resto de patrones respiratorios $(p<0.05)$. La longitud de brazada en el patrón R2 presentó diferencias significativas con los patrones respiratorios $\mathrm{R} 4$, $\mathrm{R} 5$ y $\mathrm{RL}$.

Por su parte, la tabla 3 presenta la comparación entre variables relacionadas al tamaño corporal, edad y frecuencia en las clases de natación entre los sujetos que presentaron mejores y peores desempeños técnicos.

Tabla 3. Características antropométricas de los practicantes de natación no competitiva.

\begin{tabular}{lcc}
\hline & Cluster $\mathbf{1}(\mathbf{n}=\mathbf{2 1})$ & Cluster $\mathbf{2}(\mathbf{n}=\mathbf{1 1})$ \\
\cline { 2 - 3 } & Media (DP) & Media (DP) \\
\hline IB (m²/ciclo*s) & $1.80(0.43)$ & $3.36(0.58)^{*}$ \\
Edad (años) & $39.85(11.56)$ & $31.90(13.12)$ \\
Estatura (m) & $1.68(0.11)$ & $1.77(0.04)^{*}$ \\
Peso (Kg) & $72.80(13.90)$ & $77.18(7.65)$ \\
FE (cant.) & $2.61(0.49)$ & $2.54(0.52)$ \\
\hline
\end{tabular}

Nota: $\mathrm{m}=$ metros; $\mathrm{kg}$ = kilogramos; cuantitativo = cantidad; IB = Índice de Brazadas; $\mathrm{MC}=$ Masa Corporal; FE = Frecuencia en los entrenamientos. (cluster 1 - peor desempeño técnico, cluster 2 - mejor rendimiento técnico) ${ }^{*} p<0.05$.

Se observó que la estatura media del grupo que demostró mejor calidad técnica (cluster 2) fue superior en comparación al grupo con peor desempeño técnico $(\mathrm{t}$ $=-2.88, p=0.01$ ), indicando que los nadadores con mayor estatura poseen una ventaja en la ejecución de movimientos relativos al nado crawl. Los kilogramos $(\mathrm{t}=-1.14 ; \mathrm{p}=0.26)$, la edad $(\mathrm{t}=1.76 ; \mathrm{p}=0.08)$ y la frecuencia semanal de entrenamientos $(t=0.39 ; p=0.69)$ no demuestran diferencias significativas entre los grupos mencionados.

Cuando se analizó la influencia de las variables antropométricas sobre la duración de la prueba (tabla 4), ajustadas por sexo, edad y frecuencia de entrenamiento, solamente el IMC mantuvo relación sobre el tiempo total de prueba en todos los patrones respiratorios. El peso presentó una relación significativa solamente en los patrones respiratorios R2 y R3. De las variables técnicas, la frecuencia de brazada fue la única que no obtuvo diferencias significativas. Las variables longitud de brazada e índice de brazada poseen influencias positivas en el desempeño de todos los patrones respiratorios, resultando en un $\beta$ negativo, siendo la longitud de brazada la variable que presenta mayor impacto sobre el desempeño del nado crawl, sin embargo, los ciclos de brazada aumentan el tiempo de prueba en aproximadamente 0.75 segundos en cada nuevo ciclo de brazada. 
Rev.int.med.cienc.act.fís.deporte - vol. 21 - número 82 - ISSN: 1577-0354

Tabla 4. Regresión lineal Múltiple entre variables antropométricas y técnicas con la duración de prueba.

\begin{tabular}{|c|c|c|c|c|c|}
\hline \multicolumn{6}{|c|}{ Coeficiente Beta $(\beta)$ del Patrones Respiratorios } \\
\hline & R2 & R3 & R4 & R5 & $\mathbf{R L}$ \\
\hline Peso (kg) & $0.11(0.05)^{*}$ & $0.11(0.05)^{*}$ & $0.10(0.05)$ & $0.11(0.55)$ & $0.11(0.06)$ \\
\hline Estatura (m) & $-5.47(8.19)$ & $-1.21(8.08)$ & $-2.96(8.68)$ & $-3.88(8.90)$ & $-10.28(9.0)$ \\
\hline IMC (kg/m²) & $0.40(0.15)^{*}$ & $0.36(0.14)^{*}$ & $0.35(0.16)^{*}$ & $0.39(0.16)^{*}$ & $0.49(0.16)^{*}$ \\
\hline Ciclo de Brazada (ciclos) & $0.75(0.16)^{*}$ & $0.73(0.17)^{*}$ & $0.74(0.17)^{*}$ & $0.72(0.18)^{*}$ & $0.81(0.17)^{*}$ \\
\hline Longitud de Brazada (m/ciclo) & $-3.51(1.05)^{*}$ & $-4.81(1.20)^{*}$ & $-3.83(1.19)^{*}$ & $-5.04(1.35)^{*}$ & $-6.08(1.51)^{*}$ \\
\hline Frecuencia de Brazada (ciclo/s) & $-1.47(5.66)$ & $-3.03(5.77)$ & $-5.30(5.66)$ & $-5.38(5.85)$ & $-7.70(6.06)$ \\
\hline Índice de Brazada (m²/ciclo*s) & $-2.49(0.45)^{*}$ & $-3.27(0.45)^{*}$ & $-2.94(0.47)^{*}$ & $-3.35(0.51)^{*}$ & $-3.92(0.52)^{*}$ \\
\hline
\end{tabular}

Nota: Todas las variables fueron agrupadas por sexo, edad y frecuencia de entrenamiento. * $p<0.05$. 


\section{DISCUSIÓN}

Los objetivos de este estudio fueron I) comparar el desempeño del nado crawl en nadadores aficionados con diferentes patrones respiratorios y grupos de desempeño; II) identificar las contribuciones relativas de variables antropométricas y biomecánicas sobre el desempeño del nado crawl. Como hipótesis inicial, se entiende que la menor cantidad de respiraciones por ciclos de brazada ocasionaría un mejor desempeño en el nadador, debido al efecto del arrastre activo ocasionado por los movimientos del cuerpo durante esta (Marcos Roberto Apolinário, 2010). Esta hipótesis fue comprobada por estudios desarrollados con atletas de alto rendimiento (Apolinario et al., 2012; Marcos Roberto Apolinário, 2010, 2016; Maglischo \& do Nascimento, 1999; Payton, Bartlett, Baltzopoulos, \& Coombs, 1999). En el presente estudio, esta hipótesis fue parcialmente comprobada, pues la velocidad de nado, que generalmente es considerada en las comparaciones, no sufrió influencias del patrón respiratorio. Sin embargo, se constataron diferencias sutiles en las variables que componen el ciclo del movimiento, las cuales en el proceso de enseñanza-aprendizaje se podrían desarrollar positivamente en cuanto a las ejecuciones técnicas.

En cuanto al "número de ciclos de brazadas", los patrones R2 y R3 presentaron promedios menores en relación a los demás patrones, lo que sugiere un menor gasto energético para el cumplimiento de la prueba $(25 \mathrm{~m})$. De manera similar, la "longitud de brazada", representada por la distancia recorrida durante un ciclo completo de los brazos del nadador, aumentó en los patrones respiratorios R2 y R3 en comparación con los demás, demostrando efectividad en los momentos en que se braceaba cada dos o tres respiraciones.

La mejora en el desempeño de los nadadores mediante la disminución e incluso ausencia del patrón respiratorio durante la prueba fue constatada en un estudio de Apolinario et al., (2012) quienes investigaron los efectos de diferentes patrones respiratorios en el desempeño del nado crawl, y constataron que la condición de respiración en apnea consiguió mejores velocidades medias, frecuencia y longitud de brazadas cuando se comparaban las condiciones de brazadas bilaterales, lado preferido y lado no preferido. La acción de respiración provoca efecto en las fases propulsivas y acuáticas de la brazada del nado crawl (Apolinario et al., 2012; Marcos Roberto Apolinário, 2010, 2016; de Souza Castro et al., 2005; Moré, Carpes, \& Castro, 2007).

Además, cuando los resultados presentados fueron analizados colectivamente, sugieren que la utilización de los patrones respiratorios no ocasionaron mejora en los valores de "velocidad media" y "duración", pero los nadadores aficionados emplearon mayores "frecuencias de brazadas" en los patrones respiratorios R4, $\mathrm{R} 5$, R5 y RL, lo que indica un mayor esfuerzo y gasto energético para la realización de la prueba de $25 \mathrm{~m}$ en estas condiciones. La respiración interfiere en la posición del nadador de tal forma que provoca una oscilación mayor en el momento de la rotación del tronco (Maglischo \& do Nascimento, 1999; Payton et al., 1999).

Por lo tanto se observó que para nadadores aficionados se debe priorizar la calidad en la ejecución del movimiento de la brazada con el objetivo de obtener 
una mayor longitud, pues esta característica pudo ser confirmada con los resultados del índice de brazada. El patrón respiratorio R2 obtuvo mejor índice de brazada en comparación a todos los otros cuatro ejecutados por los sujetos.

También observamos que para nadadores aficionados hay una selección natural por cuenta de los parámetros antropométricos, observándose que los sujetos con mejor ejecución técnica fueron los que poseían mayor estatura, asociándose posiblemente con una gran envergadura, la que en desplazamientos dentro del agua posibilitaría una mayor longitud de brazada.

La regresión lineal múltiple demostró que para los nadadores aficionados, la cantidad de ciclos de brazada presenta una influencia negativa sobre el rendimiento en el nado de 25 metros. Sin embargo, la longitud de brazada y el índice de brazada son variables que influencian positivamente sobre el rendimiento. A diferencia de los nadadores competitivos que necesitan mayores ciclos de brazada, los nadadores de la presente muestra presentan mejores resultados cuando la longitud de brazada es mayor, demostrando que la eficiencia del movimiento es un factor predominante en el nado crawl en esta población.

EI IB aparenta ser una medida adecuada de la técnica del nado, estableciendo que mientras mayor sea su valor mejor será su movimiento. Para nadadores de rendimiento moderado en distancias cortas y moderadas, el IB es un buen predictor de la técnica del nado crawl (Caputo et al., 2008). Demostrado en el presente estudio, en dónde cada unidad de IB mejora el tiempo de prueba en aproximadamente 3 segundos $(\beta$ medio $=3.19)$. De este modo, el índice de brazada puede ser utilizado como una variable de desempeño y eficiencia de movimiento, comprobado también por la presente investigación.

Una limitación del estudio fue la heterogeneidad en edades dentro de la muestra (18 a 60 años), esto dificultó la generalización de los resultados encontrados respecto de esta variable. Sin embargo, los resultados demostraron una similitud sobre la influencia de la respiración en poblaciones que realizan natación como deporte recreativo y no competitivo. Se sugiere que nuevos estudios sean realizados analizando diferentes modalidades de nados, poblaciones y un alcance menor de edad de los participantes y de la muestra. Como aplicaciones prácticas, se recomienda la utilización del estándar respiratorio R2 con la finalidad de obtener mejores índices de desarrollo técnico y de la planificación de procedimientos pedagógicos que busquen la ejecución de una mayor amplitud de movimiento, lo que puede, en un futuro próximo, mejorar o establecer una adquisición de movimientos más amplios.

\section{CONCLUSIONES}

Se concluye que el patrón respiratorio R2 fue más eficaz para nadadores aficionados, ya que ayudó en el equilibrio de las variables técnicas en la prueba de $25 \mathrm{~m}$. La velocidad para estos nadadores aficionados se califica en R2, más por la longitud que en la frecuencia de brazadas y el IMC fue un indicador que 
influenció negativamente en el tiempo de prueba en los diferentes patrones respiratorios, sugiriendo que el tamaño corporal puede ser un factor limitante.

\section{REFERENCIAS BIBLIOGRÁFICAS}

Apolinario, M. R., de OLIVEIRA, T. A. C., Ferreira, L. F., Basso, L., Corrêa, U. C., \& Freudenheim, A. M. (2012). Efeitos de diferentes padrões respiratórios no desempenho e na organização temporal das braçadas do nado" Crawl". Revista Brasileira de Educação Física e Esporte, 26(1), 149_ 159. doi.org/10.1590/S1807-55092012000100014

Apolinário, M R, Silva, C. G. S., Tertuliano, I. W., Oliveira, T. A. C., Marques, M. T., \& Freudenheim, A. M. (2007). Efeito da modificação estrutural na braçada do nado crawl: um estudo exploratório. XXX Simpósio Internacional de Ciências Do Esporte, 15, 248.

Apolinário, Marcos Roberto. (2010). Efeitos de diferentes padrões respiratórios no desempenho e na braçada do nado crawl. Universidade de São Paulo. doi.org/10.11606/D.39.2010.tde-03042011-220819

Apolinário, Marcos Roberto. (2016). Nado crawl : associação entre coordenação e desempenho de nadadores MARCOS ROBERTO APOLINÁRIO Nado crawl : associação entre coordenação e desempenho de nadadores. Universidade de São Paulo. doi.org/10.11606/T.39.2017.tde-13032017-085757

Caputo, F., De Lucas, R. D., Greco, C. C., \& Denadai, B. S. (2008). Características da braçada em diferentes distâncias no estilo crawl e correlações com a performance. Revista Brasileira de Ciência e Movimento, 8(3), 7-14. doi.org/10.18511/rbcm.v8i3.363

da Silva, M. C. R., de Oliveira, R. J., \& Conceição, M. I. G. (2005). Efeitos da natação sobre a independência funcional de pacientes com lesão medular. Revista Brasileira Medicina Do Esporte [Internet]. July/Ago, 11(4), 251256. doi.org/10.1590/S1517-86922005000400010

de Souza Castro, F. A., Guimarães, A. C. S., Moré, F. C., Lammerhirt, H. M., \& Maques, A. C. (2005). Cinemática do nado" crawl" sob diferentes intensidades e condições de respiração de nadadores e triatletas. Revista Brasileira de Educação Física e Esporte, 19(3), 223-232. doi.org/10.1590/S1807-55092005000300005

Guedes, D. P. (2006). Manual prático para avaliação em educação física. Editora Manole Ltda.

Hay, J. G. (1981). Biomecânica das técnicas desportivas. Interamericana.

Maglischo, E. W., \& do Nascimento, F. G. (1999). Nadando ainda mais rápido. Manole.

Marôco, J. (2014). Análise Estatística com utilização do PASW Statistics (exSPSS)(6 $6^{a}$ Edição). Pêro Pinheiro: Report Number.

Massaud, M. G., \& Côrrea, C. R. (2008). Natação na Idade Escolar. Sprint.

Moré, F. C., Carpes, F., \& Castro, F. (2007). Simetria das forças no nado crawl: influência da respiração. XII Congresso Brasileiro de Biomecânica. São Pedro, 518-523.

Payton, C. J., Bartlett, R. M., Baltzopoulos, V., \& Coombs, R. (1999). Upper extremity kinematics and body roll during preferred-side breathing and breath-holding front crawl swimming. Journal of Sports Sciences, 17(9), 
689-696. doi.org/10.1080/026404199365551

Toussaint, H. M., \& Hollander, A. P. (1994). Mechanics and energetics of front crawl swimming. In Medicine and science in aquatic sports (Vol. 39, pp. 107-116). Karger Publishers. doi.org/10.1159/000423715

Número de citas totales / Total references: 15

Número de citas propias de la revista / Journal's own references: 0

Rev.int.med.cienc.act.fís.deporte - vol. 21 - número 82 - ISSN: 1577-0354 